\title{
Dynamics of the EEG sensorimotor and alpha rhythm amplitude patterns in a training series of controlling the power platform with visual feedback
}

\author{
Sergei Makhin ${ }^{1}$, Elena Birukova ${ }^{1 *}$,Elena Chuyan ${ }^{1}$, and Oleg Kubryak ${ }^{2}$ \\ ${ }^{1}$ V.I. Vernadsky Crimean Federal University, 295007 Simferopol, Russia \\ ${ }^{2}$ Anokhin Research Institute of Normal Physiology, 125315 Moscow, Russia
}

\begin{abstract}
The study of the EEG alpha reactivity patterns in the prefrontal, central, parietal and occipital regions involved 25 healthy male subjects who participated in four training sessions of controlling the power platform with visual feedback. A significant prefrontal alpha rhythm synchronization was found which was not essentially modulated in a series of subsequent trainings. A significant desynchronization of the occipital alpha was registered for the second session which gradually increased up to the fourth session. The sensorimotor alpha rhythm didn't show regular patterns. The use of the dominant (right) hand resulted in a significant desynchronization of the parietal alpha in the right hemisphere in the third and the fourth training sessions.
\end{abstract}

\section{Introduction}

Recently, the fields of design and practical application for many types of augmenting and rehabilitation devices such as limb prostheses [1], exoskeletons [2], training and rehabilitation simulators [3] undergo a rapid development. To help develop these fields more effectively, it is necessary to accumulate quite a vast theoretical and empirical knowledge on the issues related to determining the optimal dosages and modes of motor rehabilitation, for example, when working with post-stroke patients [4]. One of the promising methods of motor rehabilitation for such patients are stabilometric simulators and their modifications. In particular, there are currently being actively studied the options of implementing the power joystick based on the ST-150 stabilometric platform with a feedback screen designed with the focus on rehabilitation of the upper limb functions. Within the framework of a comprehensive research project related to this work, it is planned to carry out an in-depth study of a number of fundamental, neuro- and psychophysiological indicators mediating the effectiveness of use of this tool. The study of the capacity for movement regulation by its force characteristics is of interest because it makes possible to register limb movements with lower amplitude which are not sufficient for the effective use of accelerometry or goniometry,

\footnotetext{
* Corresponding author: biotema@rambler.ru
} 
in order to conduct diagnostics and trainings with biological feedback when working with seriously ill patients.

The objective of the current part of the complex study was to examine the EEG amplitude modulation patterns in the alpha rhythm frequency range in a group of healthy subjects in a series of four consecutive daily trainings for controlling a stationary power joystick using left and right hands based on visual feedback. The alpha frequency EEG activity is traditionally regarded as associated with the basic indicators of activation / inhibition of relatively broad areas of the cortex. The alpha rhythm power attenuation (desynchronization) is considered as a sign of activation in the cortex areas located under corresponding electrodes, while its increase (synchronization) indicates the processes of cortex deactivation or even its active inhibition [5]. We were primarily interested in finding the patterns of activity in the cortical areas, associated with motor functions (prefrontal and central cortex), visual analysis (occipital cortex) and sensorimotor integration (parietal cortex). The analysis of the sensorimotor alpha rhythm (mu rhythm) reactivity was of particular interest due to the currently very popular research trends in the context of studying the brain system of the socalled mirror neurons, which, according to a number of authors, may underlie one's ability to implicitly understand other people's actions [6]. The effects of the mu rhythm desynchronization are often regarded as a nonspecific marker of the mirror neuron system activation (for example, [7,8]). The present research is of high relevance based on the fact that it will contribute to creating a basic model of a typical dynamics of development of the EEG reactivity patterns in healthy subjects while working with the power platform.

\section{Materials and method}

The research work was conducted on the basis of the "Experimental Physiology and Biophysics" Center in to the Department of Human and Animal Physiology and Biophysics, Taurida's Academy, V.I. Vernadsky Crimean Federal University. It involved 25 healthy right-handed male adults aged 18-25 years. The study was conducted in compliance with the modern ethical standards, according to the requirements of the local ethics commission, based on the Helsinki Declaration of the World Medical Association and GOST R 56509-2015 "Services to the population. Proper Humanitarian Research Practices".

The volunteers, after being instructed and pre-trained, participated in the four training sessions, learning to control the power platform tool for four consecutive days. During each session, they performed the following task: by means of the force applied by their right and left hands to the stationary handle (power joystick), after positioning properly the elbow, the subjects were instructed to continuously control the mark on the screen placed before them for a duration of 60 seconds within one trial, with a 1-minute rest between trials. The controlled motion of the mark corresponded to the force applied by the volunteer to the joystick, its trajectory was guided by round "targets" appearing on the screen around the perimeter of the central circle, on which, according to the instructions, the controlled mark was pointed; the mark was held on the target until it disappeared, and then center-positioned to continue the trial (according to the "Dynamic probe" algorithm implemented in the STPL software application, RU 2013610986).

In the course of performing the experimental task, there was recorded a set of electrophysiological parameters in the subjects by means of the 32-channel electroencephalograph Neuron-Spectrum-5 and its native software applications. In the context of the present study, we have analyzed the EEG alpha rhythm $(8-13 \mathrm{~Hz})$ amplitude dynamics at 12 electrodes associated with the following cortex projections: prefrontal (F3, $\mathrm{Fz}, \mathrm{F} 4)$, central $(\mathrm{C} 3, \mathrm{Cz}, \mathrm{C} 4)$, parietal (P3, Pz, P4) and occipital (O3, Oz, O4). Monopolar EEG-potentials were recorded according to the 10-20 electrode placement system. Linked earlobe reference electrodes were used. The EEG sampling rate was $250 \mathrm{~Hz}$. The signal was 
fast-Fourier transformed. The fragments of EEG with clear artifacts were excluded. Artifactfree records of no less than 30 seconds for each trial were required to include them in further statistical analysis.

The accumulated EEG amplitude values were preliminary log-transformed (using the natural logarithm) to help normalize their statistical distribution. The repeated-measures ANOVA was used to estimate the effects of such intrasubject factors as the experimental situation (SIT) (motionless relaxed state vs controlling the power joystick condition), EEG electrodes localization (LOC) (prefrontal / central / parietal / occipital) and lateralization (LAT) (left hemisphere / median line / right hemisphere) on the differences in EEG alpha amplitudes, separately for each session and different hands. The EEG amplitude differences, depending on experimental conditions (wakefulrest vs joystick manipulation), were estimated for each electrode separately by means of the method of contrasts (F-statistics).

The alpha rhythm reactivity indices were calculated for each volunteer according to the traditional scheme [9], according to the formula $k=\ln (B / A)$, where $k$ - alpha rhythm reactivity index, $B$ - alpha rhythm amplitude under joystick manipulation condition, $A-$ baseline alpha rhythm amplitude at physical rest. Positive index values indicate the alpha rhythm synchronization effect while negative - the effect of it desynchronization.

\section{Results and their discussion}

The ANOVA results showing the significance of differences in the EEG alpha rhythm amplitudes depending on the main factors and their interaction are presented in the Table 1.

Table 1. ANOVA results showing the effects of experimental situation combined with the EEG localization and lateralization on the EEG alpha rhythm when manipulating the power joystick

\begin{tabular}{|c|c|c|c|c|}
\hline $\begin{array}{c}\text { Series, } \\
\text { hand }\end{array}$ & SIT & SIT x LOC & SIT x LAT & $\begin{array}{c}\text { SIT } x \text { LOC } x \\
\text { LAT }\end{array}$ \\
\hline (1) right & $\begin{array}{l}F(1,21)=0.306 \\
\eta_{p}{ }^{2}=0.014 \\
p=0.586\end{array}$ & $\begin{array}{l}F(3,63)=5.121 \\
\eta_{p}{ }^{2}=0.200 \\
\mathbf{p}=\mathbf{0 . 0 0 3}\end{array}$ & $\begin{array}{l}F(2,42)=1.345 \\
\eta_{p}^{2}=0.060 \\
p=0,272\end{array}$ & $\begin{array}{l}F(6,126)=1.975 \\
\eta_{p}^{2}=0.086 \\
p=0.074\end{array}$ \\
\hline (1) left & $\begin{array}{l}F(1,21)=0.008 \\
\eta_{p}{ }^{2}=0.000 \\
p=0.930\end{array}$ & $\begin{array}{l}F(3,63)=5.554 \\
\eta_{p}{ }^{2}=0.209 \\
\mathbf{p}=\mathbf{0 . 0 0 2}\end{array}$ & $\begin{array}{l}F(2,42)=1.108 \\
\eta_{p}^{2}=0.050 \\
p=0.340\end{array}$ & $\begin{array}{l}F(6,126)=1.385 \\
\eta_{p}{ }^{2}=0.062 \\
p=0.226\end{array}$ \\
\hline (2) right & $\begin{array}{l}F(1,22)=0.397 \\
\eta_{p}{ }^{2}=0.018 \\
p=0.535\end{array}$ & $\begin{array}{l}\mathrm{F}(3,66)=8.375 \\
\eta_{\mathrm{p}}{ }^{2}=0.276 \\
\mathbf{p}<\mathbf{0 . 0 0 1}\end{array}$ & $\begin{array}{l}F(2,44)=0.629 \\
\eta_{p}^{2}=0.028 \\
p=0.538\end{array}$ & $\begin{array}{l}F(6,132)=1.960 \\
\eta_{p}{ }^{2}=0.082 \\
p=0.076\end{array}$ \\
\hline (2) left & $\begin{array}{l}F(1,22)=0.153 \\
\eta_{p}{ }^{2}=0.007 \\
p=0.700\end{array}$ & $\begin{array}{l}\mathrm{F}(3,66)=14.622 \\
\eta_{\mathrm{p}}{ }^{2}=0.399 \\
\mathbf{p}<\mathbf{0 . 0 0 1}\end{array}$ & $\begin{array}{l}F(2,44)=0.371 \\
\eta_{p}^{2}=0.017 \\
p=0.692\end{array}$ & $\begin{array}{l}F(6,132)=1.926 \\
\eta_{p}{ }^{2}=0.080 \\
p=0.081\end{array}$ \\
\hline (3) right & $\begin{array}{l}F(1,23)=0.601 \\
\eta_{p}{ }^{2}=0.025 \\
p=0.446\end{array}$ & $\begin{array}{l}\mathrm{F}(3,69)=13.367 \\
\eta_{\mathrm{p}}{ }^{2}=0.368 \\
\mathbf{p}<\mathbf{0 . 0 0 1}\end{array}$ & $\begin{array}{l}F(2,46)=1.636 \\
\eta_{p}^{2}=0.066 \\
p=0.206\end{array}$ & $\begin{array}{l}F(6,138)=0.761 \\
\eta_{p}{ }^{2}=0.032 \\
p=0.602\end{array}$ \\
\hline (3) left & $\begin{array}{l}\mathrm{F}(1,23)=0.111 \\
\eta_{\mathrm{p}}{ }^{2}=0.005 \\
\mathrm{p}=0.742\end{array}$ & $\begin{array}{l}F(3,69)=10.306 \\
\eta_{p}{ }^{2}=0.309 \\
\mathbf{p}<\mathbf{0 . 0 0 1}\end{array}$ & $\begin{array}{l}F(2,46)=1.403 \\
\eta_{p}{ }^{2}=0.057 \\
p=0.256\end{array}$ & $\begin{array}{l}F(6,138)=0.670 \\
\eta_{p}^{2}=0.026 \\
p=0.724\end{array}$ \\
\hline (4) right & $\begin{array}{l}F(1,23)=1.322 \\
\eta_{p}^{2}=0.054 \\
p=0.262\end{array}$ & $\begin{array}{l}\mathrm{F}(3,69)=13.074 \\
\eta_{\mathrm{p}}{ }^{2}=0.362 \\
\mathbf{p}<\mathbf{0 . 0 0 1}\end{array}$ & $\begin{array}{l}F(2,46)=2.876 \\
\eta_{p}^{2}=0.111 \\
p=0.067\end{array}$ & $\begin{array}{l}F(6,138)=0.345 \\
\eta_{p}^{2}=0.015 \\
p=0,912\end{array}$ \\
\hline (4) left & $\begin{array}{l}\mathrm{F}(1,23)=0.867 \\
\eta_{\mathrm{p}}{ }^{2}=0.036 \\
\mathrm{p}=0.362\end{array}$ & $\begin{array}{l}F(3,69)=15.807 \\
\eta_{p}{ }^{2}=0.407 \\
\mathbf{p}<\mathbf{0 . 0 0 1}\end{array}$ & $\begin{array}{l}\mathrm{F}(2,46)=2.202 \\
\eta_{\mathrm{p}}{ }^{2}=0.087 \\
\mathrm{p}=0.122\end{array}$ & $\begin{array}{l}\mathrm{F}(6,138)=1,449 \\
\eta_{\mathrm{p}}{ }^{2}=0.593 \\
\mathrm{p}=0.200\end{array}$ \\
\hline
\end{tabular}


Histograms showing the modulations in the amplitude (reactivity indices) of the alpha rhythm when performing the task of controlling the power joystick relative to the situation of relaxed wakefulness are presented below in Figure 1.

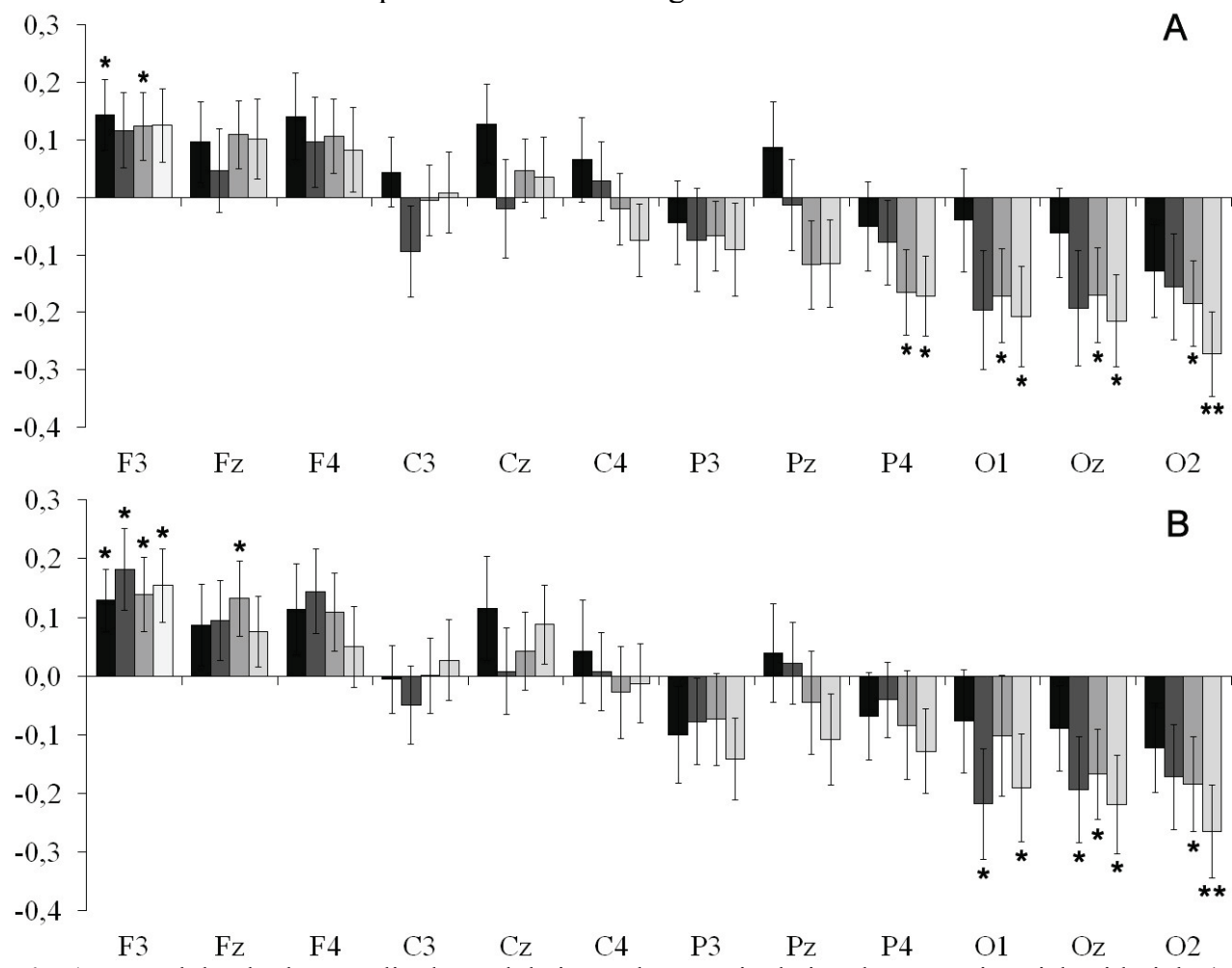

Fig. 1. EEG alpha rhythm amplitude modulations when manipulating the power joystick with right (A) and left (B) hands relative to the condition of physical rest. Y-axis indicates mean alpha rhythm reactivity index values $(\mathrm{k})$ with standard errors. $\mathrm{X}$-axis defines the EEG electrodes. The column color indicates the series number, from the darkest (1st series) to the lightest (4th series). The asterisk symbol identifies statistically significant changes of the alpha rhythm amplitudes for individual reactivity indices: $*-\mathrm{p}<0.05, * *-\mathrm{p}<0.01$.

The obtained results allowed us to identify specific patterns of the alpha rhythm reactivity when performing the experimental task of manipulating the power joystick with visual feedback, showing an overall significant alpha synchronization in prefrontal cortex regions and its pronounced desynchronization in the occipital area. The prefrontal alpha amplitude increase response doesn't show any trends of further growth or attenuation in subsequent training series. The occipital alpha desynchronization response reaches significant level already in the second series and further increases up to the fourth series.

The ANOVA results verify the statistical significance of differences in alpha rhythm amplitude change patterns observed in functionally different cortex areas, without considerable effects of lateralization associated with the use of different hands. However the more detailed analysis of the alpha rhythm reactivity under each single electrode indicates that the left prefrontal cortex gets more inhibited. Besides, the condition of task execution with the right (dominant for all volunteers) hand was accompanied by a significant decrease of alpha rhythm amplitude in the parietal region of exclusively right hemisphere, which was the case for the third and fourth training series. 
It is to be particularly noticed that we could not find any significant sensorimotor alpha $\mathrm{(mu}$ ) rhythm desynchronization response directly in the central cortex regions (at $\mathrm{C} 3, \mathrm{Cz}$ and $\mathrm{C} 4$ electrodes). This may be interpreted as an indication that the experimental task execution was not accompanied by a sustained activation of the primary motor cortex. Moreover, a significant alpha rhythm synchronization response in prefrontal regions, traditionally associated with the processes of movement planning and controlled execution, means that they undergo the effect of inhibition.

It can be hypothesized that the differences of the revealed EEG patterns from those traditionally associated with voluntary motor activity might appear to be the result of implementing quite a specific type of a stationary power joystick which is manipulated by exerting an isometric hand effort with almost no obvious amplitude. This feature may even prove useful in prospective training protocols for the motor rehabilitation of post-stroke patients with damaged cortex areas involved in the control of the upper limb movements. Quite a high involvement of the parietal-occipital cortex, associated with the processes of visual and sensorimotor integration, into learning how to effectively manipulate the power joystick, can to some extent compensate, when working with such patients, for the deficiency of direct motor control and contribute to a more rapid restoration of integral motor functions due to the formation of new effective configurations of neural connections.

\section{Conclusions}

1. A series of trainings on manipulating the power platform with visual feedback is accompanied by a significant synchronization of the prefrontal alpha rhythm, which does not considerably change in the course of the four training sessions. It is also found a significant desynchronization response in the occipital alpha rhythm, which becomes evident by the second training session and keeps growing up to the fourth session.

2. The sensorimotor alpha rhythm amplitude is not significantly modulated by executing the task of manipulating the power joystick with visual feedback.

3. Using the dominant (right) hand when manipulating the power joystick is specifically accompanied by a significant alpha rhythm desynchronization in the parietal area of the right hemisphere in the third and the fourth training series.

The research was carried out on the Scientific Experimental Physiology and Biophysics Center, Taurida Academy, V.I. Vernadsky Crimean Federal University, Russia. Supported by the Crimea Republic State Council grant from for young scientists, 2019.

\section{References}

1. M. Zecca, S. Micera, M.C. Carrozza, P. Dario, Crit Rev Biomed Eng, 45, 269-289 (2017) doi:10.1615/CritRevBiomedEng.v45.i1-6.150

2. E. Pirondini, M. Coscia, S. Marcheschi, G. Roas, F. Salsedo, A. Frisoli, M. Bergamasco, S. Micera, J Neuroeng Rehabil, 13, Article number: 9 (2016) doi:10.1186/s12984-016-0117$\mathrm{x}$

3. G.J. Kim, M. Taub, C. Creelman, C. Cahalan, M.W. O'Dell, J. Stein, Am J Occup Ther, 73, 7304345040p1-7304345040p9 (2019) doi:10.5014/ajot.2019.030908

4. S.S. Grokhovsky, O.V. Kubryak, Russian Journal of the Physical Therapy, Balneotherapy and Rehabilitation, 17, 66-71 (2018) doi:10.18821/1681-3456-2018-17-2-66-71

5. O.M. Bazanova, D. Vernon, Neurosci Biobehav Rev, 44, 94-110 (2014) doi:10.1016/j.neubiorev.2013.05.007

6. G. Rizzolatti, L. Fadiga, V. Gallese, L. Fogassi, Brain Res Cogn Brain Res, 3, 131-141 (1996) doi:10.1016/0926-6410(95)00038-0 
7. N.A. Fox, M.J. Bakermans-Kranenburg, K.H. Yoo, L.C. Bowman, E.N. Cannon, R.E. Vanderwert, P.F. Ferrari, Psychol Bull, 142, 291-313 (2016) doi:10.1037/bul0000031

8. M.A. Nacharova, S.A. Makhin, V.B. Pavlenko, Russian Journal of Physiology, 105, 311 326 (2019) doi:10.1134/S0869813919030051

9. A.I. Kaida, S.A. Makhin, E.V. Eismont, V.B. Pavlenko, Tomsk State University Journal of Biology, 45, 106-127 (2019) doi:10.17223/19988591/45/6 